\title{
PARTIAL CURVATURE STRUCTURES AND CONFORMAL GEOMETRY OF SUBMANIFOLDS
}

\author{
OLDŘICH KOWALSKI
}

\section{Introduction}

A. Fialkow [1] was the first author who carried out a systematic study of a (local) submanifold $V_{n}$ in a conformally euclidean space $V_{m}$. The conformal fundamental forms and fundamental equations introduced by him enable us to develop a theory which is quite parallel to the well-known local theory of submanifolds of a euclidean space. The following two theorems are of particular significance:

A) Conformal equivalence theorem. If $V_{m}$ and $\bar{V}_{m}$ are conformal to a euclidean space, and the corresponding conformal fundamental forms of $V_{n} \subset$ $V_{m}$ and $\bar{V}_{n} \subset \bar{V}_{m}$ are equal, then a conformal transformation exists such that $V_{m} \rightleftarrows \bar{V}_{m}$ and $V_{n} \rightleftarrows \bar{V}_{n}$.

B) Existence theorem. A subspace $V_{n}$ exists in any conformally euclidean space with preassigned conformal fundamental forms whose coefficients satisfy the fundamental equations, and is determined uniquely by a set of initial conditions.

Although Fialkow's article is original and of undisputed value, its old-fashion style and method, with awkward and tiresome calculations, make it difficult to read and understand.

The purpose of the present paper is to make the essential results of A. Fialkow more appealing, concise, and also more comprehensible to a modern mathematician.

First, we shall try to characterize a submanifold $M$ of a conformally euclidean space $N$ not by fundamental forms but by a canonical structure of its induced vector bundle $\varphi_{*} T(N)$ ( $\varphi: M \rightarrow N$ denotes the inclusion map). Roughly speaking, we shall study "Riemannian vector bundles" and, in particular "conformally euclidean vector bundles" over a manifold $M$. An important example of such a structure is the tangent bundle $T(N)$ of a Riemannian manifold $N$. For this particular case, $\mathrm{H}$. Weyl introduced the wellknown conformal curvature tensor $C$, and J. A. Schouten [8] proved his famous theorem, which can be presented in the following form: a tangent bundle $T(N)$ of a Riemannian space $N, \operatorname{dim} N \geq 4$, is a conformally euclidean vector bundle if and only if $C=0$. (A special theorem holds if $\operatorname{dim} N=3$.)

Communicated by K. Yano, August 23, 1971. 
Using an algebraic interpretation of the Weyl tensor due to I. M. Singer and J. A. Thorpe [9], the author achieved to generalize the tensor $C$ for an arbitrary Riemannian vector bundle $E \rightarrow M$ (provided with a metric connection and a solder map $j: T(M) \rightarrow E$ ), $\operatorname{dim} M \geq 3$. If $\operatorname{dim} M \geq 4$, we show that all conformally euclidean vector bundles over $M$ are characterized by the condition $C=0$. A simple result also holds for $\operatorname{dim} M=3$.

We have obtained a generalized Schouten's theorem characterizing, in an intrinsic way, local submanifolds of a conformally euclidean space, and, in our opinion, this is the true geometrical content of the work by A. Fialkow.

\section{Algebraic foundations}

Let $V$ be a $d$-dimensional real vector space with an inner product $\langle$,$\rangle , and$ let $W \subset V$ be an $m$-dimensional subspace. Denote by $\operatorname{Hom}^{c}(V, V)$ the space of all skew-symmetric endomorphisms of $V$.

$A(W, V)$-curvature structure is a bilinear map $R: W \times W \rightarrow \operatorname{Hom}^{c}(V, V)$ such that

$$
\begin{gathered}
R(u, t)=-R(t, u), \\
R(u, t) v+R(t, v) u+R(v, u) t=0, \\
\langle R(u, t) v, w\rangle=\langle R(v, w) u, t\rangle \quad \text { for any } u, t, v, w \in W .
\end{gathered}
$$

By the linearity, any $R$ can be extended to a linear map of $W \otimes W$ into $\operatorname{Hom}^{c}(V, V)$, which will be also denoted by $R$. If $W=V$, then $R$ is called a curvature structure on $V$. (As for the theory of curvature structures, see [6], [7], [9].)

Denote by $\mathscr{L}(W, V)$ the vector space of all $(W, V)$-curvature structures. We can define a natural inner product $\langle,\rangle^{*}$ on $\mathscr{L}(W, V)$ by the formula

$$
\left\langle R, R^{\prime}\right\rangle^{*}=\sum_{\alpha=1}^{d} \sum_{i, j=1}^{m}\left\langle R\left(e_{i}, e_{j}\right) e_{\alpha}, R^{\prime}\left(e_{i}, e_{j}\right) e_{\alpha}\right\rangle
$$

where $\left\{e_{1}, \cdots, e_{d}\right\}$ is an arbitrary orthonormal basis of $V$ adapted to $W$, i.e., such that $\left\{e_{1}, \cdots, e_{m}\right\}$ is an orthonormal basis of $W$.

Let us denote by $\tau: V \rightarrow W, \nu: V \rightarrow W^{\perp}$ the canonical orthogonal projections. Put

$$
\operatorname{Hom}^{s}(W, V)=\{f \in \operatorname{Hom}(W, V) ; \tau \circ f \in \operatorname{Hom}(W, W) \text { is symmetric }\} \text {. }
$$

The Ricci contraction map ricc: $\mathscr{L}(W, V) \rightarrow \operatorname{Hom}^{s}(W, V)$ is given as follows : for any $R \in \mathscr{L}(W, V)$ and $u \in W$ we put

$$
(\operatorname{ricc} R)(u)=\sum_{i=1}^{m} R\left(u, e_{i}\right) e_{i},
$$


where $\left\{e_{1}, \cdots, e_{m}\right\}$ is an orthonormal frame of $W$. (The symmetry of any map $\tau \circ \operatorname{ricc} R$ follows from (2.1)-(2.3).)

For $x, y \in V$ let us denote by $x \wedge y$ the skew-symmetric endomorphism of $V$ given by

$$
(x \wedge y) z=\langle y, z\rangle x-\langle x, z\rangle y,
$$

Define the map $h: \operatorname{Hom}^{s}(W, V) \rightarrow \mathscr{L}(W, V)$ as follows: If $A \in \operatorname{Hom}^{s}(W, V)$, then

$$
(h A)(u, t)=A u \wedge t+u \wedge A t
$$

Lemma 1. If $\operatorname{dim} W \geq 3$, then the composed map $\operatorname{ricc} \circ h: \operatorname{Hom}^{s}(W, V)$ $\rightarrow \operatorname{Hom}^{s}(W, V)$ is an isomorphism.

Proof. It suffices to show that ricc $\circ h$ is injective. Let $A \in \operatorname{Hom}^{s}(W, V)$, and (ricc $\circ h) A=0$. According to (2.5)-(2.7), we obtain

$$
\begin{aligned}
(\operatorname{ricc} \circ h) A(v) & =\sum_{i=1}^{m}(h A)\left(v, e_{i}\right) e_{i} \\
& =\sum_{i=1}^{m}\left\{\left\langle e_{i}, e_{i}\right\rangle A v-\left\langle A v, e_{i}\right\rangle e_{i}+\left\langle A e_{i}, e_{i}\right\rangle v-\left\langle v, e_{i}\right\rangle A e_{i}\right\} \\
& =(m-2) \tau(A v)+(m-1) \nu(A v)+A^{0} \cdot v=0
\end{aligned}
$$

where $A^{0}=\sum_{i=1}^{m}\left\langle A e_{i}, e_{i}\right\rangle$. In particular,

$$
\tau(A v)=-\frac{A^{0}}{m-2} \tau v, \quad A^{0}=\sum_{i=1}^{m}\left\langle\tau\left(A e_{i}\right), e_{i}\right\rangle=-\frac{m A^{0}}{m-2} .
$$

Thus $A^{0}=0 \Rightarrow A v=0, v \in W \Rightarrow A=0$.

Lemma 2. $\operatorname{Im}(h)$ is orthogonal to $\operatorname{Ker}($ ricc) in $\mathscr{L}(W, V)$.

Proof. Let $A \in \mathrm{Hom}^{s}(W, V), R=h A, R^{\prime} \in \mathrm{Ker}$ (ricc). According to (2.4)-(2.7) and (2.1)-(2.3), we have

$$
\begin{aligned}
\left\langle R, R^{\prime}\right\rangle^{*}= & \sum_{\alpha=1}^{d} \sum_{i, j=1}^{m}\left\langle\left\langle e_{j}, e_{\alpha}\right\rangle A e_{i}-\left\langle A e_{i}, e_{\alpha}\right\rangle e_{j}+\left\langle A e_{j}, e_{\alpha}\right\rangle e_{i}\right. \\
& \left.-\left\langle e_{i}, e_{\alpha}\right\rangle A e_{j}, R^{\prime}\left(e_{i}, e_{j}\right) e_{\alpha}\right\rangle \\
= & \sum_{\alpha=1}^{d} \sum_{i, j=1}^{m}\left\langle\delta_{j \alpha} A e_{i}-\delta_{i \alpha} A e_{j}, R^{\prime}\left(e_{i}, e_{j}\right) e_{\alpha}\right\rangle \\
+ & +\sum_{\alpha=1}^{d} \sum_{i, j=1}^{m}\left\{\left\langle A e_{j}, e_{\alpha}\right\rangle\left\langle R^{\prime}\left(e_{i}, e_{j}\right) e_{\alpha}, e_{i}\right\rangle\right. \\
& \left.-\left\langle A e_{i}, e_{\alpha}\right\rangle\left\langle R^{\prime}\left(e_{i}, e_{j}\right) e_{\alpha}, e_{j}\right\rangle\right\} \\
= & 2 \sum_{i=1}^{m}\left\langle\left(\operatorname{ricc} R^{\prime}\right)\left(e_{i}\right), A e_{i}\right\rangle+2 \sum_{j=1}^{m}\left\langle\left(\operatorname{ricc} R^{\prime}\right)\left(e_{j}\right), A e_{j}\right\rangle=0
\end{aligned}
$$


Put $\mathscr{L}_{w}(W, V)=\operatorname{Ker}\left(\right.$ ricc), and let $j: \mathscr{L}_{w}(W, V) \rightarrow \mathscr{L}(W, V)$ be the canonical injection.

Theorem 1.1. Let $W \subset V, \operatorname{dim} W \geq 3$. Then there is a unique linear map $C: \mathscr{L}(W, V) \rightarrow \mathscr{L}_{w}(W, V)$, called the Weyl map, and a unique linear map $D: \mathscr{L}(W, V) \rightarrow \mathrm{Hom}^{s}(W, V)$, called the deviation map, such that the following commutative diagram with two exact sequences holds:

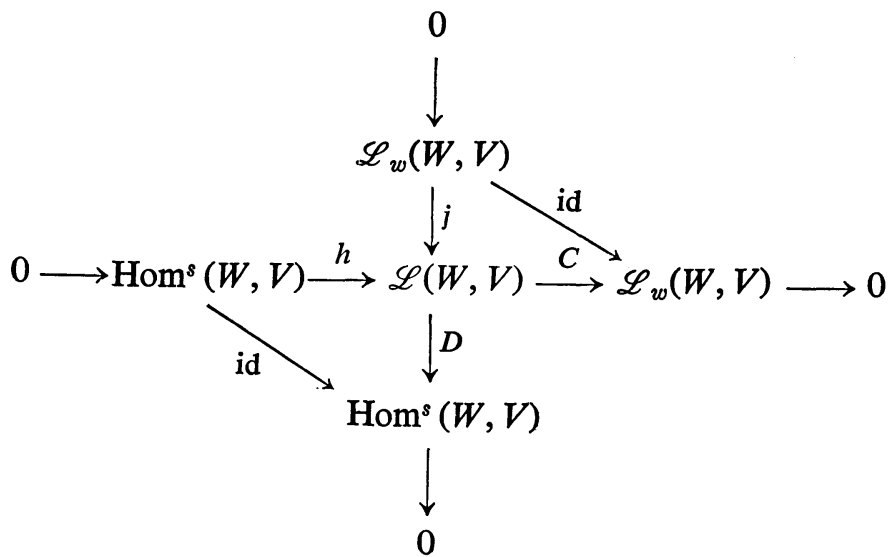

Moreover, the decomposition $\mathscr{L}(W, V)=\operatorname{Im}(h) \oplus \mathscr{L}_{w}(W, V)$ is orthogonal; thus the Weyl map $C$ is the orthogonal projection of the space $\mathscr{L}(W, V)$ onto its subspace $\mathscr{L}_{w}(W, V)$.

Proof. According to Lemma 1 the map ricc is surjective, and we have an exact sequence

$$
0 \longrightarrow \mathscr{L}_{w}(W, V) \stackrel{j}{\longrightarrow} \mathscr{L}(W, V) \stackrel{\text { ricc }}{\longrightarrow} \operatorname{Hom}^{s}(W, V) \longrightarrow 0 .
$$

Further, $h$ is injective and hence $\operatorname{dim} \operatorname{Im}(h)=\operatorname{dim} \operatorname{Hom}^{s}(W, V)$. Consequently, $\operatorname{dim} \mathscr{L}_{w}(W, V)+\operatorname{dim} \operatorname{Im}(h)=\operatorname{dim} \mathscr{L}(W, V)$. According to Lemma 2, $\mathscr{L}_{w}(W, V) \perp \operatorname{Im}(h)$, and hence follows the orthogonal decomposition $\mathscr{L}(W, V)=\operatorname{Im}(h) \oplus \mathscr{L}_{w}(W, V)$. The rest is obvious.

Remark 1. The statements of Theorem 1.1 are known in the case $W=V$. I. M. Singer and J. A. Thorpe [9] proved that the classical Weyl tensor arises from the orthogonal projection $C: \mathscr{L}(V, V) \rightarrow \mathscr{L}_{w}(V, V)$. The existence of maps $h$ and $D$ and the orthogonality of $\operatorname{Im}(h)$ and $\mathscr{L}_{w}(V, V)$ were found by K. Nomizu [7].

Remark 2. The composed map $\tau \circ D$ is an algebraic equivalent for the "deviation tensor" introduced by A. Fialkow [1]. Hence the name "deviation map" for $D$.

Conventions. Put $\mathscr{L}_{c}(W, V)=\operatorname{Im}(h)$. The elements of $\mathscr{L}_{c}(W, V)$ will be called conformally euclidean $(W, V)$-structures. The elements of the factor 
space $\mathscr{C}(W, V)=\mathscr{L}(W, V) / \mathscr{L}_{c}(W, V)$ will be called conformal $(W, V)$ curvature structures. Obviously, if $\operatorname{dim} W \geq 3$, then we have a canonical isomorphism $C^{*}: \mathscr{C}(W, V) \rightarrow \mathscr{L}_{w}(W, V)$.

Two elements $R, R^{\prime} \in \mathscr{L}(W, V)$ will be said to be conformally equivalent, if they belong to the same element of $\mathscr{C}(W, V)$. Finally, we shall briefly write $\mathscr{L}(V), \mathscr{L}_{c}(V), \mathscr{C}(V)$ for $\mathscr{L}(V, V), \mathscr{L}_{c}(V, V), \mathscr{C}(V, V)$.

We can express the maps $C, D$ explicitly. Let $R \in \mathscr{L}(W, V)$. Similarly as in the proof of Lemma 1 we obtain

$$
(\operatorname{ricc} R)(v)=\operatorname{ricc}(h A)(v)=(m-2) \tau(A v)+(m-1) \nu(A v)+A^{0} \cdot v,
$$

where $A=D R$. Hence follows

$$
D R=\frac{\tau \circ \operatorname{ricc} R}{m-2}+\frac{\nu \circ \operatorname{ricc} R}{m-1}-\frac{(\operatorname{sc} R) \cdot I}{2(m-1)(m-2)},
$$

where sc $R=\operatorname{trace}(\tau \circ \operatorname{ricc} R$ ) is the "scalar curvature" of $R$. Further, according to (2.8) we have $C R=R-(h \circ D) R$ and hence

$$
\begin{aligned}
C R(u, t)= & R(u, t)-D R(u) \wedge t-u \wedge D R(t) \\
= & R(u, t)-\frac{1}{m-2}[\tau \circ \operatorname{ricc} R(u) \wedge t-\tau \circ \operatorname{ricc} R(t) \wedge u] \\
& +\frac{(\operatorname{sc} R) \cdot u \wedge t}{(m-1)(m-2)} \\
& \quad-[\nu \circ \operatorname{ricc} R(u) \wedge t-\nu \circ \operatorname{ricc} R(t) \wedge u] /(m-1) .
\end{aligned}
$$

Here the last term occurs only if $W \neq V$.

In the following we shall study relations between the spaces $\mathscr{L}_{c}(W, V)$ and $\mathscr{L}_{c}(V)$.

The inclusion map $i: W \rightarrow V$ induces a canonical surjection $\tilde{i}: \operatorname{Hom}^{s}(V, V)$

$\rightarrow \operatorname{Hom}^{s}(W, V)$ and a map $i^{*}: \mathscr{L}(V) \rightarrow \mathscr{L}(W, V)$.

Lemma 3. The following commutative diagram holds:

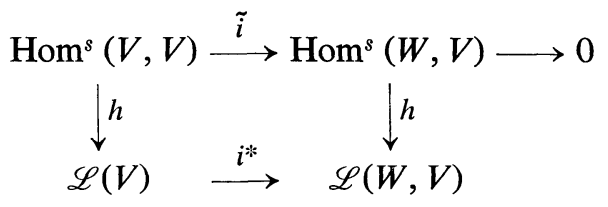

Consequently, $i^{*}\left(\mathscr{L}_{c}(V)\right)=\mathscr{L}_{c}(W, V)$.

Proof is obvious.

Thus the restriction of a conformally euclidean curvature structure $R^{\prime}: V \times$ $V \rightarrow \operatorname{Hom}^{c}(V, V)$ to the subset $W \times W$ is a conformally euclidean $(W, V)$ curvature structure. Further, for $\operatorname{dim} W \geq 3$, the restriction map $i^{*}: \mathscr{L}(V) \rightarrow$ $\mathscr{L}(W, V)$ induces a canonical map of $\mathscr{C}(V)$ into $\mathscr{C}(W, V)$. 
Let $p: \operatorname{Hom}^{s}(V, V) \rightarrow \operatorname{Hom}^{s}\left(W^{\perp}, W^{\perp}\right)$ be given as follows: for $A \epsilon$ $\operatorname{Hom}^{s}(V, V)$ we put $p A=\nu \cdot A \cdot i^{\perp}$, where $i^{\perp}: W^{\perp} \rightarrow V$ is the inclusion map.

Lemma 4. There are unique maps $\tilde{Q}: \operatorname{Hom}^{s}(W, V) \rightarrow \operatorname{Hom}^{s}(V, V)$ and $\tilde{S}: \operatorname{Hom}^{s}\left(W^{\perp}, W^{\perp}\right) \rightarrow \operatorname{Hom}^{s}(V, V)$ such that the following commutative diagram with two exact sequences holds:

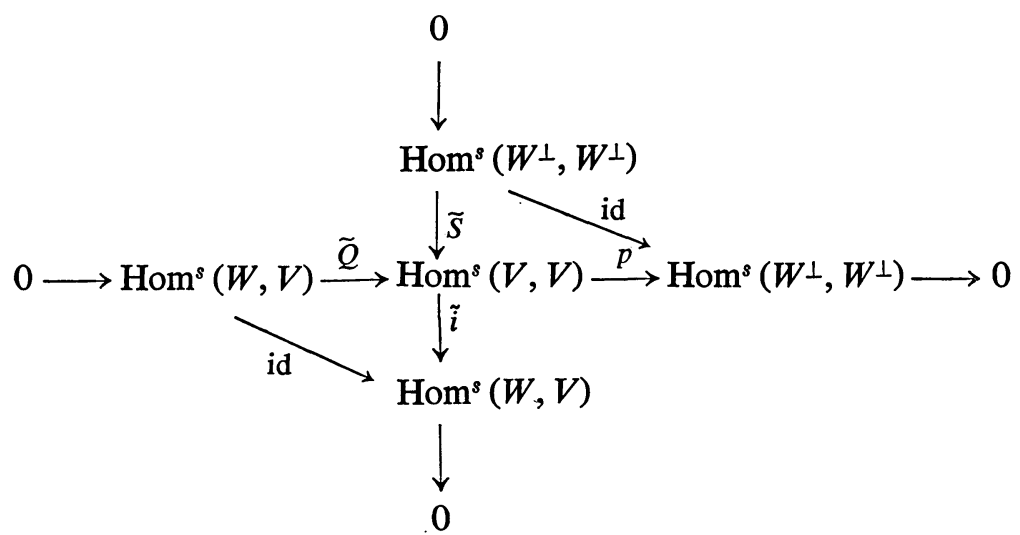

Proof. Let us define $\tilde{Q}$ and $\tilde{S}$ by the following formulas:

$$
\begin{gathered}
\langle\tilde{Q} A(x), y\rangle=\langle A(\tau x), y\rangle+\langle x, A(\tau y)\rangle-\langle\tau x, A(\tau y)\rangle, \\
\langle\tilde{S} B(x), y\rangle=\langle B(\nu x), \nu y\rangle
\end{gathered}
$$

for $x, y \in V, A \in \operatorname{Hom}^{s}(W, V), B \in \operatorname{Hom}^{s}\left(W^{\perp}, W^{\perp}\right)$. We can see easily that

a) $\tilde{Q} A, \tilde{S} A \in \operatorname{Hom}^{s}(V, V)$,

b) the commutativity of (2.12) and the exactness of both sequences hold. For uniqueness suppose that there is a map

$$
Q^{\prime}: \operatorname{Hom}^{s}(W, V) \rightarrow \operatorname{Hom}^{s}(V, V) \text { such that } \tilde{i} \circ Q^{\prime}=0, \quad p \circ Q^{\prime}=0 \text {. }
$$

Then for any $A \in \operatorname{Hom}^{s}(W, V)$ we can write

$$
\begin{aligned}
\left\langle Q^{\prime} A(x), y\right\rangle= & \left\langle Q^{\prime} A(\tau x), y\right\rangle+\left\langle Q^{\prime} A(\tau y), \nu x\right\rangle+\left\langle Q^{\prime} A(\nu x), \nu y\right\rangle \\
= & \left\langle\left(\tilde{i} \circ Q^{\prime}\right) A(\tau x), y\right\rangle+\left\langle\left(\tilde{i} \circ Q^{\prime}\right) A(\tau y), \nu x\right\rangle \\
& +\left\langle\left(p \circ Q^{\prime}\right) A(\nu x), \nu y\right\rangle=0 .
\end{aligned}
$$

Hence $Q^{\prime}$ is a null map and $\tilde{Q}$ is unique. The proof for $\tilde{S}$ is quite similar.

Theorem 1.2. Let $W \subset V, \operatorname{dim} W \geq 3$. Then there is a unique map $Q: \mathscr{L}_{c}(W, V) \rightarrow \mathscr{L}_{c}(V)$, called canonical completion, such that the following commutative diagram with two exact sequences holds: 


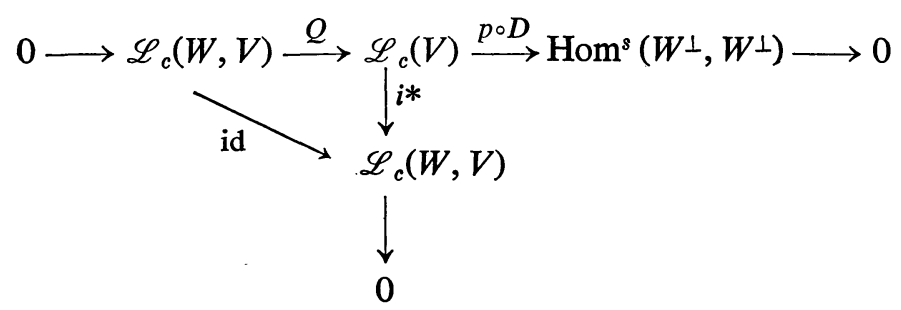

Proof. Transform the lower part of the commutative diagram (2.12) by the isomorphisms $D: \mathscr{L}_{c}(V) \rightarrow \operatorname{Hom}^{s}(V, V), D: \mathscr{L}_{c}(W, V) \rightarrow \operatorname{Hom}^{s}(W, V)$ (see Theorem 1.1) and use Lemma 3.

In particular, we have $Q=h \circ \tilde{Q} \circ D$.

In case $\operatorname{dim} W^{\perp}$ is also greater than or equal to 3 , we can obtain a stronger result. Let $h^{\nu}: \operatorname{Hom}^{s}\left(W^{\perp}, W^{\perp}\right) \rightarrow \mathscr{L}\left(W^{\perp}\right)$ be the corresponding map (2.7), and let $p^{*}: \mathscr{L}(V) \rightarrow \mathscr{L}\left(W^{\perp}\right)$ be the canonical restriction map. (More precisely, if $R \in \mathscr{L}(V), x, y, z \in W^{\perp}$, we put $\left(p^{*} R\right)(x, y)(z)=\nu[R(x, y)(z)]$.)

Lemma 5. The following commutative diagram holds independently of $\operatorname{dim} W^{\perp}$ :

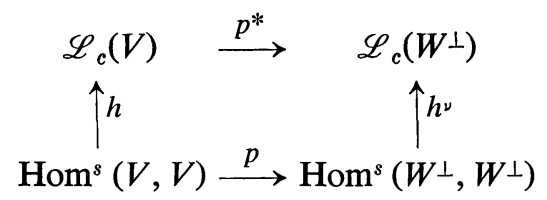

This lemma can be proved by a direct calculation.

Now, if $\operatorname{dim} W^{\perp} \geq 3$, then the map $h^{\nu}$ is an isomorphism, and its inverse is the deviation map $D^{\nu}$.

Theorem 1.3. Let $W \subset V, \operatorname{dim} W \geq 3, \operatorname{dim} W^{\perp} \geq 3$. Then there are unique linear maps $Q: \mathscr{L}_{c}(W, V) \rightarrow \mathscr{L}_{c}(V), S: \mathscr{L}_{c}\left(W^{\perp}\right) \rightarrow \mathscr{L}_{c}(V)$ (canonical completions) such that the following commutative diagram with two exact sequences holds:

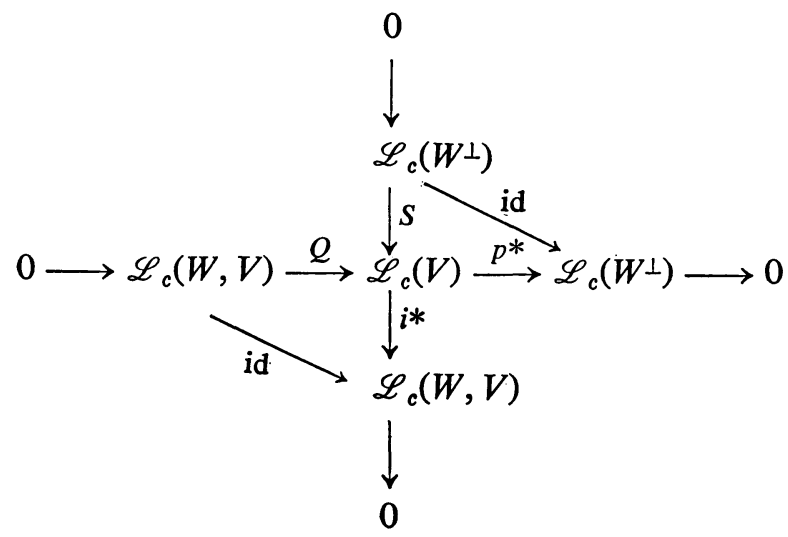


Moreover, $Q$ is identical with the map introduced in Theorem 1.2, and the decomposition $\mathscr{L}_{c}(V)=\operatorname{Im}(Q) \oplus \operatorname{Im}(S)$ is orthogonal with respect to the inner product on $\mathscr{L}(V)$.

Proof. We obtain all statements concerning diagram (2.17) by transforming (2.12) by the deviation maps $D: \mathscr{L}_{c}(V) \rightarrow \operatorname{Hom}^{s}(V, V), D: \mathscr{L}_{c}(W, V) \rightarrow$ $\operatorname{Hom}^{s}(W, V), D^{\nu}: \mathscr{L}_{c}\left(W^{\perp}\right) \rightarrow \operatorname{Hom}^{s}\left(W^{\perp}, W^{\perp}\right)$, which all are isomorphisms. We use also Lemma 3 and Lemma 5 here. The orthogonality property is easy.

\section{Generalized Schouten's theorem}

By a Riemannian vector bundle $E(A, \nabla) \rightarrow M$ we shall mean a real vector bundle $E \rightarrow M$ over a real manifold $M$, provided with a fibre metric $A$ and a metric connection $\nabla$. (Cf. [2, pp. 116-117].)

Recall that a linear connection $\nabla$ in $E$ is metric if and only if

$$
U A(X, Y)=A\left(\nabla_{U} X, Y\right)+A\left(X, \nabla_{U} Y\right)
$$

for any vector field $U$ and any sections $X, Y: M \rightarrow E$.

Any vector subbundle $F$ of $E(A, \nabla)$ is canonically a Riemannian vector bundle $F\left(A, \nabla^{F}\right)$, where $\nabla^{F}$ denotes the orthogonal projection of the connection $\nabla$ into $F$ (cf. [4] for details). $F\left(A, \nabla^{F}\right)$ will be called a Riemannian subbundle of $E(A, \nabla)$.

Let $\operatorname{dim} E \geq \operatorname{dim} M$, and let $j: T(M) \rightarrow E$ be a fixed bundle injection ("solder map"). The Riemannian vector bundle $E(A, \nabla) \rightarrow M$ will be called $j$-adapted to $M$ if $\nabla$ is torsion-free with respect to $j$, i.e.,

$$
\nabla_{U}(j T)-\nabla_{T}(j U)-j[U, T]=0
$$

for any vector fields $U, T$ of $M$.

Finally, a soldered Riemannian vector bundle $E(A, \nabla) \rightarrow M$ is a Riemannian vector bundle $E(A, \nabla)$ together with a fixed solder map $j: T(M) \rightarrow E$ such that $E$ is $j$-adapted to $M$.

Example. Let $\varphi: M \rightarrow N$ be an immersion of a manifold $M$ into a Riemannian manifold $N$. Then the induced bundle $\varphi_{*} T(N)$ is canonically a soldered Riemannian vector bundle $E(A, \nabla)$ over $M$. Here $A, \nabla$ are determined by the Riemannian metric and the Riemannian connection of $N$ respectively.

As a rule, we shall identify $T(M)$ with $j T(M) . T(M)$ is canonically a Riemannian subbundle $T(M)\left(A, \nabla^{\tau}\right)$ of $E(A, \nabla)$, called the tangent subbundle of $E$. Here $A$ is a Riemannian metric on $M$ and, according to (3.2), $\nabla^{\tau}$ is the corresponding Riemannian connection on $M$ (see [4] for details). The Riemannian subbundle $T(M)^{\perp}\left(A, \nabla^{\nu}\right)$, where $\nabla^{\nu}$ is the orthogonal projection of $\nabla$ into $T(M)^{\perp}$, is called the normal subbundle of $E$.

We shall introduce canonical projections

$$
\tau: E \rightarrow T(M), \quad \nu: E \rightarrow T(M)^{\perp} .
$$


Recall that the curvature transformation of a Riemannian vector bundle $E(A, \nabla)$ is the bundle morphism $\mathscr{R}: T(M) \otimes T(M) \rightarrow$ Hom $(E, E)$ defined by

$$
\mathscr{R}(U, T) X=\nabla_{U} \nabla_{T} X-\nabla_{T} \nabla_{U} X-\nabla_{[U, T]} X
$$

for any two vector fields $U, T$ on $M$ and any section $X: M \rightarrow E$.

Let now $E(A, \nabla)$ be soldered to $M$. For any point $p \in M$ and any two vectors $u, t \in T_{p}(M)$ we have $\mathscr{R}(u, t) \in \operatorname{Hom}^{c}\left(E_{p}, E_{p}\right)$. Consequently, we obtain a $\left(T_{p}(M), E_{p}\right)$-curvature structure $\mathscr{R}_{p}$ on any fibre $E_{p}$ of $E$. According to Theorem 1.1 we can introduce the following definition.

Definition 1. Let $E(A, \nabla) \rightarrow M$ be a soldered Riemannian vector bundle with $\operatorname{dim} M \geq 3$, and denote by $\mathscr{R}$ its curvature transformation. Then the bundle morphism $C \mathscr{R}: T(M) \otimes T(M) \rightarrow \operatorname{Hom}(E, E)$ is called the Weyl transformation of $E(A, D)$, and the bundle morphism $D \mathscr{R}: T(M) \rightarrow E$ is called the deviation transformation of $E(A, \nabla)$.

We shall briefly write $\mathscr{C}, \mathscr{D}$ for $C \mathscr{R}, D \mathscr{R}$, and recall that

$$
\mathscr{R}(U, T)=\mathscr{C}(U, T)+\mathscr{D}(U) \wedge T+U \wedge \mathscr{D}(T)
$$

for any two vector fields $U, T$ on $M$, where the exterior product $\wedge$ is taken with respect to the metric $A$. (See formulas (2.6) and (2.10).)

Proposition 1. Let $E(A, \nabla) \rightarrow M$ be a soldered Riemannian vector bundle with $\operatorname{dim} M \geq 4$ such that its Weyl transformation $\mathscr{C}$ vanishes. Then a "Codazzi equation"

$$
\left(\nabla_{U} \mathscr{D}\right)(V)-\left(\nabla_{V} \mathscr{D}\right)(U)=0
$$

holds for any two vector fields $U, V$ on $M$. Here $\left(\nabla_{U} \mathscr{D}\right)(V)=\nabla_{U} \mathscr{D}(V)-$ $\mathscr{D}\left(\nabla_{U}^{\tau} V\right)$ by definition.

Proof. Define the covariant derivative $\nabla_{T} \mathscr{R}$ of $\mathscr{R}$ by

$$
\begin{aligned}
\left(\nabla_{T} \mathscr{R}\right)(U, V) X= & \nabla_{T}[\mathscr{R}(U, V) X]-\mathscr{R}\left(\nabla_{T}^{\tau} U, V\right) X \\
& -\mathscr{R}\left(U, \nabla_{T}^{\tau} V\right) X-\mathscr{R}(U, V)\left(\nabla_{T} X\right) .
\end{aligned}
$$

Then the second Bianchi identity

$$
\left(\nabla_{T} \mathscr{R}\right)(U, V)+\left(\nabla_{U} \mathscr{R}\right)(V, T)+\left(\nabla_{V} \mathscr{R}\right)(T, U)=0
$$

holds (cf. [4]) for any vector fields $T, U, V$ on $M$. According to (3.5), we have $\mathscr{R}(U, T)=\mathscr{D}(U) \wedge T+U \wedge \mathscr{D}(T)$, and (3.7) implies

$$
(\Delta \mathscr{D})(T, U) \wedge V+(\Delta \mathscr{D})(U, V) \wedge T+(\Delta \mathscr{D})(V, T) \wedge U=0,
$$

where $(\Delta \mathscr{D})(U, V)=\left(\nabla_{U} \mathscr{D}\right)(V)-\left(\nabla_{V} \mathscr{D}\right)(U)$.

Now by applying [5, Lemma 4] to the case where $d=1, A^{1}=$ identity map, and $\Delta_{1}=\Delta \mathscr{D}$, we obtain $\triangle \mathscr{D}=0$. 
Remark. Consider the case $E \equiv T(M)$. Then our Proposition is an immediate consequence of [7, Proposition 4]. This statement represents a decisive step of any proof of the classical Schouten's theorem.

Let $E(A, \nabla)$ be a soldered Riemannian vector bundle over $M$, and define the first normal tensor $P$ on $M$ as a bundle morphism $P: T(M) \otimes T(M) \rightarrow E$ determined by

$$
P(U, T)=\nabla_{U} T-\nabla_{U}^{\tau} T=\nu\left(\nabla_{U} T\right)
$$

for any vector fileds $U, T$ on $M$ (cf. [4]). Because $V$ is torsion-free, $P(U, T)=$ $P(U \otimes T)$ is symmetric.

Now the mean curvature vector field $\mu$ of $E(A, \nabla)$ is a section $\mu: M \rightarrow E$ defined at any $p \in M$ by the formula

$$
\mu(p)=\frac{1}{m} \sum_{i=1}^{m} P\left(e_{i}, e_{i}\right)
$$

where $\left\{e_{1}, \cdots, e_{m}\right\}$ is an orthonormal frame of $T_{p}(M)$ (cf. [1]).

Let now $\operatorname{dim} E>\operatorname{dim} M$. The conformal measure tensor $G: T(M) \otimes T(M)$ $\rightarrow E$ is determined by

$$
G(U, T)=P(U, T)-A(U, T) \mu .
$$

A point $p \in M$ is said to be umbilical in $E$ if $G$ vanishes at $p$.

At any non-umbilical point $p \in M$ we define the gauge function $\alpha$ by the formula

$$
e^{2 \alpha}=\frac{1}{m} \sum_{i=1}^{m} \sum_{j=1}^{m} A\left(G\left(e_{i}, e_{j}\right), G\left(e_{i}, e_{j}\right)\right)
$$

where $\left\{e_{1}, \cdots, e_{m}\right\}$ is an arbitrary orthonormal frame of $T_{p}(M)$ (see [1, p. 327]).

We shall say that $E(A, \nabla) \rightarrow M$ is $\nabla$-connected if at any point $p \in M$ we have $E_{p}=\bigcup_{k=1}^{\infty} S_{p}^{k}$, where $S_{p}^{k}$ denotes the osculating space of order $k$ at $p$. (Cf. [4, p. 682].)

Two soldered Riemannian vector bundles $E(A, \nabla), E^{\prime}\left(A^{\prime}, \nabla^{\prime}\right)$ over the same manifold $M$ are said to be equivalent and denoted by $E^{\prime} \cong E$ if there is a bundle isomorphism $\phi: E \rightarrow E^{\prime}$ (called an equivalence) such that

(i) $\Phi$ is an identity map on the common tangent subbundle $T(M)$,

(ii) $\Phi^{*} \nabla^{\prime}=\nabla$, and $\Phi^{*} A^{\prime}=A$.

Proposition 2. The only equivalence of a $\nabla$-connected bundle $E(A, \nabla)$ onto itself is the identity map. Consequently, if $E(A, \nabla) \cong E^{\prime}\left(A^{\prime}, \nabla^{\prime}\right)$, and $E(A, \nabla)$ is $\nabla$-connected, then there is a unique (canonical) equivalence $\Phi: E(A, \nabla) \rightarrow$ $E^{\prime}\left(A^{\prime}, \nabla^{\prime}\right)$.

Proof. By induction on the order $k \geq 1$. 
Proposition 3. Let $\varphi: M \rightarrow N$ be an immersion of a manifold $M$ into a Riemannian manifold $N$, and $F: N \rightarrow N$ an isometric transformation. Then the soldered Riemannian bundles $\varphi_{*} T(M),(F \circ \varphi)_{*} T(M)$ are canonically equivalent.

Proof is obvious and therefore omitted.

Definition 2. Two soldered Riemannian vector bundles $E(A, \nabla), E\left(A^{\prime}, \nabla^{\prime}\right)$ with the same underlying vector bundle $\pi: E \rightarrow M$ are said to be conformally equivalent and denoted by $E\left(A^{\prime}, \nabla^{\prime}\right)=(\psi, \xi) \square E(A, \nabla), A^{\prime} \stackrel{c}{\sim} A$, if there is a real function $\psi$ on $M$, called the transition function, and a section $\xi: M$ $\rightarrow E$, called the transition section, such that

$$
\begin{gathered}
A^{\prime}=\left(e^{2 \psi} \circ \pi\right) \cdot A \quad \text { on } E, \\
\nabla_{U}^{\prime} X=\nabla_{U} X+A(\xi, X) U+A(\xi, U) X-A(U, X) \xi
\end{gathered}
$$

for any vector field $U$ on $M$ and any section $X: M \rightarrow E$.

Definition 3. Two soldered Riemannian vector bundles $E(A, \nabla), E^{\prime}\left(A^{\prime}, \nabla^{\prime}\right)$ are said to be conformally equivalent and denoted by $E(A, \nabla) \stackrel{c}{\sim} E^{\prime}\left(A^{\prime}, \nabla^{\prime}\right)$ if there is a bundle $E\left(A^{*}, \nabla^{*}\right)$ which is equivalent to $E^{\prime}\left(A^{\prime}, \nabla^{\prime}\right)$ and conformally equivalent to $E(A, \nabla)$.

Remark. One can see easily that the relation $\stackrel{c}{\sim}$ actually is an equivalence relation.

Proposition 4. Let $\varphi: M \rightarrow N$ be an immersion of a manifold $M$ into a Riemannian manifold $N$. If $F: N \rightarrow N$ is a conformal transformation of $N$ into itself, then the induced bundles $\varphi_{*} T(M)$ and $(F \circ \varphi)_{*} T(N)$ are conformally equivalent in a canonical way.

Proof. Obviously, it suffices to show the following:

Let $\varphi: M \rightarrow N$ be an immersion of $M$ into a Riemannian manifold $N$ with the metric $A$, and denote by $N^{\prime}$ the same manifold $N$ provided with a conformally equivalent metric $A^{\prime}=e^{2 \psi} \cdot A$. Then the induced bundles $\varphi_{*} T(N)$, $\varphi_{*} T\left(N^{\prime}\right)$ are canonically conformally equivalent.

Let $\nabla, \nabla^{\prime}$ be Riemannian connections of $N, N^{\prime}$ respectively, and let $\xi$ denote the gradient of $\psi$ with respect to $A$. Then

$$
\begin{aligned}
\nabla_{X}^{\prime} Y & =\nabla_{X} Y+(Y \psi) X+(X \psi) Y-A(X, Y) \xi \\
& =\nabla_{X} Y+A(\xi, Y) X+A(\xi, X) Y-A(X, Y) \xi
\end{aligned}
$$

for any two vector fileds $X, Y$ on $N$. (See [6, Proposition 7] for instance). Hence $\varphi_{*} T\left(N^{\prime}\right)=(\psi, \xi) \square \varphi_{*} T(N)$. q.e.d.

Let $V$ be a vector space with the inner product $A$. Consider the map $L_{A}: V$ $\rightarrow$ Hom $(V \otimes V, V)$ given by

$$
L_{A}(w)(t, u)=A(w, t) u+A(w, u) t-A(u, t) w
$$

for $t, u, w \in V$. 
Lemma 1. The map $L_{A}$ is injective.

Proof. Let $\left\{e_{1}, \cdots, e_{n}\right\}$ be an orthonormal frame of $V$. Then $\sum_{i=1}^{n} L(w)\left(e_{i}, e_{i}\right)$ $=(2-n) w$, and hence follows our assertion for $n \neq 2$. If $n=2$, then $L(w)\left(e_{1}, e_{2}\right)=A\left(w, e_{1}\right) e_{2}+A\left(w, e_{2}\right) e_{1}$ and $L(w)=0$, so that $w=0$.

Proposition 5. Let $E\left(A^{\prime}, \nabla^{\prime}\right)=(\psi, \xi) \square E(A, \nabla)$ and denote by $P, P^{\prime}, \mu, \mu^{\prime}$ the corresponding first normal tensors and mean curvature vector fields. Then

$$
\begin{gathered}
\nabla_{U}^{\prime \tau} T=\nabla_{U}^{\tau} T+L_{A}(\tau \circ \xi)(U, T), \\
P^{\prime}(U, T)=P(U, T)+L_{A}(\nu \circ \xi)(U, T)=P(U, T)-A(U, T)(\nu \circ \xi), \\
\tau \circ \xi=\operatorname{grad}_{A} \psi(=\text { gradient of } \psi \text { with respect to } A \text { on } M), \\
\nu \circ \xi=\mu-e^{2 \psi} \cdot \mu^{\prime} .
\end{gathered}
$$

Proof. Let us consider the tangent and normal parts of the formula (3.13) for the case $X=T=$ vector fields. Then we have (3.16) and (3.17). Further, replacing the manifold $N$ by the manifold $M$ in (3.14) gives $\nabla_{U}^{\tau} T=\nabla_{U}^{\tau} T+$ $L_{A}\left(\operatorname{grad}_{A} \psi\right)(U, T)$. Using (3.16) and Lemma 1 we obtain (3.18). Finally, (3.19) follows from (3.9) and (3.17).

Proposition 6. If $E(A, \nabla)$ is a soldered Riemannian vector bundle with $\psi, \xi$ satisfying (3.18), then the operation $(\psi, \xi) \square E(A, \nabla)$ determines a soldered Riemannian vector bundle $E\left(A^{\prime}, \nabla^{\prime}\right) \stackrel{c}{\sim} E(A, \nabla)$.

Proof is easy and therefore omitted. (See also the proof of Proposition 12.)

Proposition 7. Let $E\left(A^{\prime}, \nabla^{\prime}\right)=(\psi, \xi) \square E(A, \nabla)$, and $\operatorname{dim} E \geq \operatorname{dim} M$, and denote by $G^{\prime}, G, \alpha^{\prime}, \alpha$ the corresponding conformal measure tensors and gauge functions. Then

$$
\begin{gathered}
G^{\prime}(U, T)=G(U, T) \quad(U, T \in T(M)), \\
\alpha^{\prime}=\alpha-\psi .
\end{gathered}
$$

Proof is obvious from (3.10), (3.11), (3.17), (3.19).

A soldered bundle $E(A, \nabla) \rightarrow M$ will be said to be conformally $\nabla$-connected if any $E^{\prime}\left(A^{\prime}, \nabla^{\prime}\right) \stackrel{c}{\sim} E(A, \nabla)$ is $\nabla^{\prime}$-connected. Obviously, if $\operatorname{dim} E=\operatorname{dim} M$, then $E(A, \nabla)$ is conformally $\nabla$-connected. For $\operatorname{dim} E \geq \operatorname{dim} M$ we have the following criterion:

Proposition 8. Let $E(A, \nabla)$ be a soldered Riemannian vector bundle such that $\operatorname{dim} E \geq \operatorname{dim} M$, and let $\mu$ be its mean curvature vector field. Then $E(A, \nabla)$ is conformally $\nabla$-connected if and only if the bundle $E\left(A, \nabla^{0}\right)=(0, \mu) \square E(A, \nabla)$ is $\nabla^{0}$-connected.

Proof. Let $\mu^{0}$ denote the mean curvature vector field of $E\left(A, \nabla^{0}\right)$. From (3.19) we get $\mu^{0}=\mu-\nu \circ \mu=0$. Let $E\left(A^{\prime}, \nabla^{\prime}\right) \stackrel{c}{\sim} E\left(A, \nabla^{0}\right), E\left(A, \nabla^{0}\right)=$ $\left(\psi^{\prime}, \xi^{\prime}\right) \square E\left(A^{\prime}, \nabla^{\prime}\right)$. Then $\nu \circ \xi^{\prime}=\mu^{\prime}-e^{2 \psi^{\prime}} \mu^{0}=\mu^{\prime}$ and $\nabla_{U}^{0} X=\nabla_{U}^{\prime} X+$ 
$L_{A^{\prime}}\left(\xi^{\prime}\right)(U, X)=\nabla_{U}^{\prime} X+L_{A^{\prime}}\left(\operatorname{grad} \psi^{\prime}+\mu^{\prime}\right)(U, X)$. Put $S^{\prime k}=\cup_{p \in M} S_{p}^{\prime k},{ }^{0} S^{k}=$ $\bigcup_{p \in M}{ }^{0} S_{p}^{k}$, where $S_{p}^{\prime k},{ }^{0} S_{p}^{k}$ denote the osculating spaces of order $k$ in $E\left(A^{\prime}, \nabla^{\prime}\right)$, $E\left(A, \nabla^{0}\right)$ respectively. Obviously, ${ }^{0} S^{1} \equiv T(M) \subset S^{\prime 1}$. Suppose that ${ }^{0} S^{k} \subset S^{\prime k}$ for a fixed $k \geq 1$. Let $X^{(k)}: M \rightarrow E$ be a section such that $\operatorname{Im}\left(X^{(k)}\right) \subset{ }^{0} S^{k}$. Then for $U \in T_{p}(M), p \in M$, we obtain $\nabla_{U}^{0} X^{(k)}=\nabla_{U}^{\prime} X^{(k)}+L_{A^{\prime}}\left(\operatorname{grad} \psi^{\prime}+\mu^{\prime}\right)_{p}$ $\cdot\left(U, X^{(k)}\right)$. Because $\nabla_{U}^{\prime} X^{(k)} \in S_{p}^{\prime k+1},\left(\operatorname{grad} \psi^{\prime}+\mu^{\prime}\right)_{p} \in S_{p}^{\prime 2}, X_{p}^{(k)} \in S_{p}^{\prime k}$, we get $\nabla_{U}^{0} X^{(k)} \in S_{p}^{\prime k+1}$. Hence follows ${ }^{0} S_{p}^{k+1} \subset S_{p}^{\prime k+1}$.

We have proved by induction that $\bigcup_{k=1}^{\infty}{ }^{0} S^{k} \subset \bigcup_{k=1}^{\infty} S^{k}$. Because $E\left(A, \nabla^{0}\right)$ is $\nabla^{0}$-connected, $E\left(A^{\prime}, \nabla^{\prime}\right)$ is $\nabla^{\prime}$-connected. The rest is trivial.

Proposition 9. Let $E\left(A^{\prime}, \nabla^{\prime}\right)=(\psi, \xi) \square E(A, \nabla)$, and denote by $\mathscr{R}, \mathscr{R}^{\prime}$ the curvature transformations of $E(A, \nabla), E\left(A^{\prime}, \nabla^{\prime}\right)$ respectively. Then

$$
\mathscr{R}^{\prime}(U, T)=\mathscr{R}(U, T)-\mathscr{H}(U) \wedge T-U \wedge \mathscr{H}(T),
$$

where $\mathscr{H}: T(M) \rightarrow E$ is a bundle morphism given by

$$
\mathscr{H}(U)=\nabla_{U} \xi-A(U, \xi) \xi+\frac{1}{2} A(\xi, \xi) U,
$$

and the exterior product $\wedge$ is taken with respect to the metric $A$.

Proof. If follows from (3.4) and (3.13) by direct calculation.

Now from (3.18) and (3.23) we can see that $\mathscr{H} \in \operatorname{Hom}^{s}(T(M), E)$. Hence we get

Proposition 10. If $E(A, \nabla), E\left(A^{\prime}, \nabla^{\prime}\right)$ are conformally equivalent, then the corresponding curvature transformations $\mathscr{R}, \mathscr{R}^{\prime}$, as $\left(T_{p}(M), E_{p}\right)$-curvature structures at each point $p \in M$, are conformally equivalent. In particular, if $\operatorname{dim} M$ $\geq 3$, then the corresponding Weyl transformations are equal, i.e., $\mathscr{C}=\mathscr{C}^{\prime}$.

A soldered Riemannian vector bundle $E(A, \nabla) \rightarrow M$ will be said to be

a) euclidean, if $\mathscr{R}=0$,

b) conformally euclidean, if it is conformally equivalent to a euclidean bundle,

c) locally conformally euclidean, if it is conformally euclidean over a neighborhood of any point $p \in M$.

The following assertion is a consequence of Proposition 4 :

Proposition 11. Let $\varphi: M \rightarrow N$ be an immersion of a manifold $M$ into a euclidean (conformally euclidean) space. Then the induced bundle $\varphi_{*} T(N) \rightarrow$ $M$ is euclidean (conformally euclidean).

Now for euclidean bundles we have a converse global theorem, which follows from [4, Theorem 1].

Theorem A. Let $E(A, D) \rightarrow M$ be a $\nabla$-connected euclidean bundle over a simply connected manifold $M, \operatorname{dim} E=d$. Then there is exactly one immersion $\varphi: M \rightarrow \boldsymbol{E}^{d}$ of $\boldsymbol{M}$ into a d-dimensional euclidean space $\boldsymbol{E}^{d}$ such that

(i) $\varphi_{*} T\left(E^{d}\right) \cong E(A, \nabla)$,

(ii) with respect to the canonical equivalence, a preassigned orthonormal frame $\left\{f_{1}, \cdots, f_{d}\right\}$ of $\boldsymbol{T}\left(\boldsymbol{E}^{d}\right)$ corresponds to a preassigned orthonormal frame 
$\left\{e_{1}, \cdots, e_{d}\right\}$ of $E$. In particular, the immersion $\varphi$ is isometric with respect to the Riemannian metric $A$ induced on $M$ by $E(A, \nabla)$.

In the following, we shall derive some (local) existence theorems of the above type for locally conformally euclidean bundles, and also characterize these bundles by the quantities $\mathscr{C}$ and $\mathscr{D}$.

Let $E(A, \nabla) \rightarrow M, \operatorname{dim} M \geq 3$, be conformally euclidean, i.e., $\mathscr{C}=0$. Let $\xi: M \rightarrow E$ be a transition section from $E(A, \nabla)$ to a euclidean vector bundle $E\left(A^{\prime}, \nabla^{\prime}\right)$. Then $\mathscr{R}^{\prime}=0$ and (3.5), (3.22), (3.23) imply

$$
\nabla_{U} \xi-A(U, \xi) \xi+\frac{1}{2} A(\xi, \xi) U=\mathscr{D}(U),
$$

where $\mathscr{D}$ is the deviation transformation of $E(A, \nabla)$. So $\xi$ satisfies a system of linear partial differential equations of the first order:

$$
\nabla_{U} \xi=A(U, \xi) \xi-\frac{1}{2} A(\xi, \xi) U+\mathscr{D}(U) .
$$

By covariant differentiations we obtain (using (3.5))

$$
\left[\nabla_{U}, \nabla_{V}\right] \xi-\nabla_{[U, V]} \xi=\mathscr{R}(U, V) \xi-\left(\nabla_{V} \mathscr{D}\right)(U)+\left(\nabla_{U} \mathscr{D}\right)(V)
$$

and hence the Codazzi equation (3.6) holds. Moreover, the system (3.24) is completely integrable. In other words, for any element $z \in E$ and any sufficiently small neighborhood $U$ of $p=\pi(z)$ in $M$ there is a unique local section $\xi_{z}: U \rightarrow E$ satisfying (3.24) and the initial condition $\xi(p)=z$.

Proposition 12. Let $E(A, \nabla)$ be a soldered Riemannian vector bundle over a simply connected manifold $M, \operatorname{dim} M \geq 3$, with vanishing Weyl transformation $\mathscr{C}$. Then any section $\xi: M \rightarrow E$ satisfying (3.24) is a transition section from $E(A, \nabla)$ to a euclidean vector bundle $E\left(A^{\prime}, \nabla^{\prime}\right)$. Moreover, if the Codazzi equation (3.6) holds, then the system (3.24) is completely integrable.

Proof. We show first that a transition function $\psi$ exists. From (3.24) we obtain $A\left(\nabla_{U} \xi, V\right)=A\left(\nabla_{V} \xi, U\right)$ for any two vector fields $U, V$ on $M$ and hence follows easily

$$
U A(\tau \circ \xi, V)-V A(\tau \circ \xi, U)-A(\tau \circ \xi,[U, V])=0,
$$

which means that $\tau \circ \xi$ is locally a gradient. Because $M$ is simply connected, we have $\tau \circ \xi=\operatorname{grad}_{A} \psi, \psi: M \rightarrow \boldsymbol{R}$ being determined uniquely exactly up to an additive constant. Put $A^{\prime}=e^{2 \psi} \cdot A$. If we define $\nabla^{\prime}$ by formula (3.13), we can see easily, using the relation $\tau \circ \xi=\operatorname{grad}_{A} \psi$, that $\nabla^{\prime}$ is metric with respect to $A^{\prime}$. Further, we show immediately that $\nabla^{\prime}$ is torsion-free. Finally, (3.22)(3.24) and (3.5) yield $\mathscr{R}^{\prime}=\mathscr{C}=0$, i.e., $E\left(A^{\prime}, \nabla^{\prime}\right)$ is euclidean. The rest is obvious.

Proposition 13. Let $E(A, \nabla) \rightarrow M, \operatorname{dim} M \geq 3$, be a locally conformally euclidean vector bundle, $p \in M$ a fixed point, $w \in T_{p}(M)$ and $n \in T_{p}(M)^{\perp}$ given vectors, and $\lambda$ a real number. Then there are a neighborhood $U$ of $p$ in $M$ 
and a unique euclidean vector bundle $\left.E\left(A^{\prime}, \nabla^{\prime}\right)\right|_{U}$ over $U$ such that
a) $\left.\left.E\left(A^{\prime}, \nabla^{\prime}\right)\right|_{U} \stackrel{c}{\sim} E(A, \nabla)\right|_{U}$
b) $A^{\prime}=e^{2 \lambda} \cdot A, \nabla^{\prime \tau}=\nabla^{\tau}+L_{A}(w)$ at the point $p$,
c) $\mu_{p}^{\prime}=n$.

Proof. We shall construct the quantities $\psi$ and $\xi$. The condition $b$ ) is equivalent to $\psi(p)=\lambda$ and $(\tau \circ \xi)_{p}=\left(\operatorname{grad}_{A} \psi\right)_{p}=w$ (Lemma 1). Further, c) implies $(\nu \circ \xi)_{p}=\mu_{p}-e^{2 \lambda} \cdot n$ (see (3.19)). Thus the initial values $\xi_{p}, \psi(p)$ of the required transition quantities are determined. Now let us take a sufficiently small simply connected neighborhood $U \ni p$ and apply Proposition 12 .

Convention. We shall denote the euclidean bundle constructed in Proposition 13 by $E_{U}^{0}(A, \nabla, p, w, n, \lambda)$.

Remark that if $E(A, \nabla)$ is conformally $\nabla$-connected, then any bundle $\left.E\left(A^{\prime}, \nabla^{\prime}\right)\right|_{U}=E_{U}^{0}(A, \nabla, p, w, n, \lambda)$ is $\nabla^{\prime}$-connected.

Corollary. Let $N, \operatorname{dim} N \geq 3$, be a conformally euclidean space with the metric $G$, and $q \in N$ a fixed point. Then there is exactly one euclidean metric $G^{\prime}$ on a neighborhood $W \ni q$ such that

a) $G^{\prime} \stackrel{c}{\sim} G$ on $W$,

b) $G^{\prime}=G$ and $\nabla^{\prime}=\nabla$ at q, i.e., $G^{\prime}$ is osculating to $G$ at $q$.

Convention. The corresponding euclidean space will be denoted by $N^{0}(q, W)$.

Theorem 2.1 (Existence theorem). Let $E(A, \nabla) \rightarrow M, \operatorname{dim} M \geq 3$, be a locally conformally euclidean vector bundle which is conformally $\nabla$-connected. Let $N$ be a conformally euclidean space, $\operatorname{dim} N=\operatorname{dim} E$. Further, let $p \in M$, $q \in N$ be fixed points, $f: E_{p} \rightarrow T_{q}(N)$ a prescribed homothety with coefficient $e^{2 \lambda}$, and $w \in T_{p}(M), n \in T_{p}(M)^{\perp}$ given vectors. Then there is a unique imbedding $\varphi$ of a neighborhood $U(p) \subset M$ into a neighborhood $W(q) \subset N$ such that

(i ) $\varphi(p)=q$, (ii) $E_{U}^{0}(A, \nabla, p, w, n, \lambda) \cong \varphi_{*} T\left(N^{0}(q, W)\right)$,

(iii) the canonical equivalence map $\Phi$ coincides with $f$ on $E_{p}$. Moreover,

a) the imbedding $\varphi: U \rightarrow W$ is conformal,

b) $(d \varphi)_{p}$ is a homothety with coefficient $e^{2 \lambda}$,

c) $\left(\varphi^{*} \nabla^{N}\right)^{\tau}=\nabla^{\tau}+L_{A}(w)$ at $p,\left(\nabla^{N}\right.$ : the Riemannian connection of $\left.N\right)$,

d) $f(n)$ is the mean curvature vector of $\varphi(U) \subset N$ at $q$.

Proof. See Proposition 13, its Corollary, and Theorem A.

Remark. We can also state an existence theorem without the requirement that $E$ be conformally $\nabla$-connected, but the formulation of such a theorem would be a little awkward (Cf. [4, Theorem 1].)

Theorem 2.2 (Equivalence theorem). Let $\varphi, \varphi^{\prime}: M \rightarrow N$ be two immersions of a manifold $M, \operatorname{dim} M \geq 3$, into a conformally euclidean space $N$, such that $\varphi_{*} T(N) \stackrel{c}{\sim} \varphi_{*}^{\prime} T(N)$. Then for any point $p \in M$ there are a neighborhood $U \ni p$ in $M$, a neighborhood $W \supset \varphi(U)$ in $N$ and a conformal map $F: W \rightarrow$ $N$ such that $\varphi^{\prime}=F \circ \varphi$ on $U$. 
Outline of the proof. Let us apply Proposition 13 to the bundle $T(N)(G, \nabla)$ $\rightarrow N$ at the points $q=\varphi(p), q^{\prime}=\varphi^{\prime}(p)$. So we can construct a euclidean metric $G_{1} \stackrel{c}{\sim} G$ in a neighborhood $W_{1}(q)$ and a euclidean metric $G_{2} \stackrel{c}{\sim} G$ in a neighborhood $W_{2}\left(q^{\prime}\right)$ with preassigned initial conditions. Now we can suitably choose these initial conditions so that the induced euclidean bundles $E\left(\varphi^{*} G_{1}, \varphi^{*} \nabla_{1}\right), E^{\prime}\left(\varphi^{*} G_{2}, \varphi^{*} \nabla_{2}\right)$ over $V=\varphi^{-1}\left(W_{1}\right) \cap \varphi^{\prime-1}\left(W_{2}\right) \subset M$ satisfy $\varphi^{*} G_{1}=\varphi^{*} G_{2},\left(\varphi^{*} \nabla_{1}\right)^{\tau}=\left(\varphi^{*} \nabla_{2}\right)^{\tau}, \mu_{1}=\mu_{2}=0$ at the point $p$. Hence according to the uniqueness part of Proposition 13, we have $E\left(\varphi^{*} G_{1}, \varphi^{*} \nabla_{1}\right) \cong$ $E^{\prime}\left(\varphi^{\prime *} G_{2}, \varphi^{\prime *} \nabla_{2}\right)$ over a neighborhood $U \subset V$. From Theorem 1, [4], we can see that there is an isometry $F$ of $W \subset W_{1}(q)$ (provided with the metric $G_{1}$ ) into $W_{2}\left(q^{\prime}\right)$ (provided with the metric $G_{2}$ ) such that $\varphi^{\prime}=F \circ \varphi$ on $U$. If we return to the original metric $G$ on $N$, then $F: W \rightarrow W_{2}$ is conformal.

Theorem 2.3 (Generalized Schouten's theorem). Let $E(A, \nabla) \rightarrow M$, $\operatorname{dim} M \geq 3$, be a soldered Riemannian vector bundle with the Weyl transformation $\mathscr{C}$ and the deviation transformation $\mathscr{D}$.

I. For $\operatorname{dim} M \geq 4, E$ is locally conformally euclidean if and only if $\mathscr{C}=0$.

II. For $\operatorname{dim} M=3, E$ is locally conformally euclidean if and only if $\mathscr{C}=0$ and $\left(\nabla_{U} \mathscr{D}\right)(V)-\left(\nabla_{V} \mathscr{D}\right)(U)=0$.

Proof. We use Proposition 12 and Proposition 1.

Remark. The condition $\mathscr{C}=0$ in the statement II cannot be neglected if $E \neq T(M)$.

Theorem 2.4 (Second existence theorem). Let $E(A, \nabla) \rightarrow M, \operatorname{dim} M \geq 3$, be a $\nabla$-connected, locally conformally euclidean vector bundle, and $p \in M a$ fixed point. Then there is a neighborhood $U \ni p$, a conformally euclidean space $N, \operatorname{dim} N=\operatorname{dim} E$, and an imbedding $\varphi: U \rightarrow N$ such that

(i) $\left.\varphi_{*} T(N) \cong E(A, \nabla)\right|_{U}$,

(ii) the curvature transformation $\mathscr{R}^{N}$ of $N$ along $\varphi(U)$ is the canonical completion of the curvature transformation $\mathscr{R}$ of $E$ over $U: \mathscr{R}^{N}=Q \mathscr{R}$.

Outline of the proof. 1) We construct a euclidean bundle $E\left(A^{\prime}, \nabla^{\prime}\right) \stackrel{c}{\sim}$ $E(A, \nabla)$ over $U \ni p$.

2) We take an imbedding $\varphi$ of $U$ into a euclidean space $N$ such that $\varphi_{*} T(N) \cong E\left(A^{\prime}, \nabla^{\prime}\right)$ over $U$.

3) We construct a tubular neighborhood $W$ of $\varphi(U)$ in $N$ and a convenient local coordinate system in $W$ using the "normal exponential map" $\exp ^{\perp}: T(\varphi(U))^{\perp} \rightarrow N$.

4) Put $E(A, \nabla)=(\psi, \xi) \square E\left(A^{\prime}, \nabla^{\prime}\right)$. We shall seek a function $\subseteq$ on $W$ which is a transition function from the given euclidean metric to a required conformally euclidean metric in $N$.

The requirement (i) implies $\mathbb{S}=\psi$ and $\operatorname{grad} \subseteq=\xi$ along $\varphi(U)$, i.e., gives the values of $\mathfrak{S}$, the first partial derivatives of $\mathbb{S}$ and some second partial derivatives of $\mathbb{\subseteq}$ along $\varphi(U)$. 
Now the requirement $\mathscr{R}^{N}=Q \mathscr{R}$ can be expressed in the form $\mathscr{H}^{N}=\tilde{Q} \mathscr{H}$ (see corresponding relations (3.22), (3.23)), and the last condition determines uniquely the remaining second partial derivatives of $\subseteq$ along $\varphi(U)$. Then it is easy to give a coordinate expression for $\subseteq$. q.e.d.

A soldered Riemannian vector bundle $E(A, \nabla) \rightarrow M$ will be said to be umbilical (or non-umbilical) if all points of $M$ are umbilical (or non-umbilical) in $E$.

Proposition 14 (cf. [1, pp. 426-427]). Let $E(A, \nabla) \rightarrow M$ be an umbilical, locally conformally euclidean vector bundle. Then the tangent subbundle $T(M)\left(A, \nabla^{\tau}\right)$ is locally conformally euclidean, and consequently, so is the manifold $M$.

Proof. If $\operatorname{dim} M \leq 2$, the assertion is trivial. Let now $\operatorname{dim} M \geq 3$. Then the bundle $E\left(A, \nabla^{\prime}\right)=(0, \mu) \square E(A, \nabla)$ satisfies $P^{\prime}(U, T)=G(U, T)=0$ for $U, T \in T(M)$, according to (3.10), (3.17) and due to the fact that $E$ is umbilical. From (3.8) we obtain $\nabla^{\prime}=\nabla^{\prime \tau}=\nabla^{\tau}$ on $T(M)$ and hence $\mathscr{R}^{\tau}(U, T) V$ $=\mathscr{R}^{\prime}(U, T) V$ for $U, T, V \in T(M)$, where $\mathscr{R}^{\tau}$ denotes the curvature transformation of $T(M)\left(A, \nabla^{\tau}\right)$. Consequently, ricc $\mathscr{R}^{\tau}=\operatorname{ricc} \mathscr{R}^{\prime}, \operatorname{sc} \mathscr{R}^{\tau}=\operatorname{sc} \mathscr{R}^{\prime}, \mathscr{D}^{\tau}=$ $\mathscr{D}^{\prime}$ and $\mathscr{C}^{\tau}(U, T) V=\mathscr{C}^{\prime}(U, T) V$ for $U, T, V \in T(M)$ (see formulas (2.5), (2.9), (2.10)). Now the assertion is a consequence of Theorem 2.3.

Proposition 15. Let $E(A, \nabla) \rightarrow M$ be a non-umbilical soldered Riemannian vector bundle. Then there is exactly one bundle $E\left(A^{0}, \nabla^{0}\right) \stackrel{c}{\sim} E(A, \nabla)$ with vanishing mean curvature vector field $\mu^{\prime}$ and gauge function $\alpha^{\prime}$.

Proof. According to (3.19), (3.21) it suffices to put $E\left(A^{0}, \nabla^{0}\right)=$ $\left(\alpha, \operatorname{grad}_{A} \alpha+\mu\right) \square E(A, \nabla)$. (Cf. [1, p. 333].)

We shall call $E\left(A^{0}, \nabla^{0}\right)$ the Fialkow bundle of $E(A, \nabla)$.

Proposition 16. I. A non-umbilical bundle $E(A, \nabla)$ is conformally $\nabla$-connected if and only if its Fialkow bundle $E\left(A^{0}, \nabla^{0}\right)$ is $\nabla^{0}$-connected.

II. Two non-umbilical bundles $E(A, \nabla), E^{\prime}\left(A^{\prime}, \nabla^{\prime}\right)$ are conformally equivalent if and only if their Fialkow bundles are equivalent.

Proof. The argument for Part I is quite similar to the proof of Proposition 8. Part II is a consequence of Proposition 15.

Now we can introduce the canonical graduation, the normal tensors, the fundamental forms and the Bompiani forms of a Fialkow bundle similar to those for a euclidean bundle in [4], [5]. Due to the strength of the generalized Schouten's theorem one can develop all the theory of A. Fialkow [1] in a more appealing compact form. The details will be published elsewhere.

\section{References}

[1] A. Fialkow, Conformal differential geometry of a subspace, Trans. Amer. Math. Soc. 56 (1944) 309-433.

[2] S. Kobayashi \& K. Nomizu, Foundations of differential geometry, Vol. I, Interscience, New York. 1963.

[ 3 ] — Foundations of differential geometry, Vol. II, Interscience, New York, 1969. 
[4] O. Kowalski, Immersions of Riemannian manifolds with a given normal bundle structure. I, Czechoslovak Math. J. 19 (94) (1969) 676-695.

[5] - Type numbers in metric differential geometry of higher order, J. Differential Geometry 7 (1972) 403-421.

[6] R. S. Kulkarni, Curvature structures and conformal transformations, J. Differential Geometry 3 (1969) 493-510.

[ 7 ] K. Nomizu, On the decomposition of generalized curvature tensor fields, preprint.

[ 8 ] J. A. Schouten, Über die konforme Abbildung n-dimensionaler Mannigfaltigkeiten mit quadratischer Massbestimmung auf eine Mannigfaltigkeit mit euclidischer Massbestimmung, Math. Z. 11 (1921) 58-88.

[9] I. M. Singer \& J. A. Thorpe, The curvature of 4-dimensional Einstein spaces, Global Analysis, Papers in Honor of K. Kodaira, Princeton University Press, Princeton, 1969, 355-365.

Charles University, Prague 